\title{
Study of Asymmetric Traffic Influence on IEEE 802.11 WLAN Family, Enhancement Proposals
}

\author{
Elena Lopez-Aguilera, Jordi Casademont, Josep Cotrina \\ Telematics Department - Technological University of Catalonia (UPC)
}

\section{INTRODUCTION}

Since 1997 when the IEEE defined the first standard IEEE 802.11 for wireless local area networks, it has evolved a lot. The former IEEE 802.11 worked at $2.4 \mathrm{GHz}$ and at data rates of 1 and $2 \mathrm{Mbps}$; later it appeared IEEE 802.11b that using the same frequency got $11 \mathrm{Mbps}$. IEEE 802.11a was developed next, this one changed its working frequency to 5 $\mathrm{GHz}$ reaching $54 \mathrm{Mbps}$. However, the change of frequency represented a drawback on interoperability with older equipment, so it gave place to the development of IEEE $802.11 \mathrm{~g}$ that got the $54 \mathrm{Mbps}$ working again at $2.4 \mathrm{GHz}$. Finally, in September 2003 a new working group has begun to work in order to develop IEEE 802.11 n that should get more than $100 \mathrm{Mbps}$.

Many studies appeared on different aspects of IEEE 802.11 [1]-[5]. An aspect to have in mind is that most WLAN are used as the access network of a set of computers to the local intranet or towards global Internet, and in few occasions, the traffic is between two components of the same BSS. This issue is responsible that most WLANs are infrastructure networks.

All the previous cited papers study IEEE 802.11 performance considering a symmetric traffic distribution between all computers of the Basic Service Set (BSS), and we can see that this hypothesis. differ from the majority of installed WLAN. This paper is focused, then, in a new study of the relevant parameters of WLAN performance considering IEEE 802.11 networks that are in a situation of asymmetric traffic, where the Access Point (AP) transmits much more than user stations (US). This situation is very different from the symmetric one, where all the stations have the same traffic load.

\section{SYSTEM EVALUATION UNDER SYMMETRIC AND ASYMMETRIC TRAFFIC}

In order to analyse the IEEE 802.11 performance, we use a software simulation tool implemented in UPC. This software has been validated comparing the results with those published in [1], under identical simulation conditions. Our simulation program, written in $\mathrm{C}++$ programming language, follows all the IEEE 802.11 protocol details. It emulates as closely as possible the real operation of each transmitting station.

The standard IEEE $802.11 \mathrm{~g}$ has been chosen to realize this study. Simulation environment consists in one BSS composed of $1 \mathrm{AP}$ and 10 US. The stations transmit data packets with constant payload size of 1023 bytes, and the time between consecutive arrivals follows an exponential distribution function. All US are under coverage area. Hidden terminal situation and transmission errors are not considered. Finally, the contention window takes the values of $\mathrm{CW}_{\min }=32$ and $\mathrm{CW}_{\max }=1024$ and no backoff is done between two consecutive data frames from the same station.

For the study of the asymmetric situation, we consider the AP transmitting the same amount of traffic than the 10 US altogether. Taking an example, this means that for a global normalized offered load of 0.6 , the AP offers 0.3 and user stations offer 0.03 each one. On the other hand, in order to evaluate the protocol performance under symmetric traffic, we assume that in this scenario the AP and user stations are transmitting the same amount of traffic. Taking the same example of previous paragraph, for 0.6 of offered traffic, AP and US are transmitting $0.6 / 11=0.054$ each one.

An interesting parameter to analyse is the queue delay, defined as the time that a packet ready to be transmitted is delayed until it becomes the first in its transmission queue. Obviously, the AP average queue delay under asymmetric traffic is higher than under symmetric traffic, since the AP in the first case is more loaded than in the second (Fig. 1). Considering an asymmetric traffic situation, the AP has more delay than the US at higher loads, for the different transmission rates. For $1 \mathrm{Mbps}$ with an offered load of 0.8 , the AP has 24 times more delay than user stations.

On the other hand, user station queue delay is lower under asymmetric traffic because US are offering less traffic.

Analyzing the throughput, shown in Fig. 2, for low loaded networks the network throughput does not take difference between symmetric and asymmetric traffic, but it does in high loaded networks. For a transmission rate of 1 Mbps, with an offered load of 0.8 , we obtain a throughput of 0.757 in presence of symmetric traffic, whereas with asymmetric traffic we get 0.798 . In this case, an asymmetric traffic situation enables more throughput because the number of collisions per transmitted packet is significantly lower, and also the percentage of time in which no station is transmitting, because all of them are in backoff state. The reason of this behavior is that in symmetric traffic, and at high load, all 11 stations will 
always have a queued packet for transmitting, and therefore each packet will enter in a backoff state, and the probability of collisions is also high because all stations want to access to the medium. In the case of asymmetric traffic, the AP will always have its queue full, but in many occasions US will have no packet to be transmitted, so there is less probability to collide and also less time in backoff state.

Table I. Parameters used in the simulations

\begin{tabular}{|c|c|c|}
\hline & $\begin{array}{c}802.11 \mathrm{~g} \\
(802.11 \mathrm{~b} \\
\text { compatible) }\end{array}$ & $\begin{array}{c}802.11 \mathrm{~g} \\
\text { (ERP-OFDM) }\end{array}$ \\
\hline Transmission data rate (Mbps) & $1,2,5.5,11$ & $\begin{array}{c}6,9,12,18 \\
24,36,48,54\end{array}$ \\
\hline MAC header & \multicolumn{2}{|c|}{34 bytes } \\
\hline ACK & \multicolumn{2}{|c|}{14 bytes } \\
\hline Propagation time & \multicolumn{2}{|c|}{$1 \mu \mathrm{s}$} \\
\hline Long PHY Preamble & $144 \mu \mathrm{s}$ & \multirow{2}{*}{$16 \mu \mathrm{s}$} \\
\hline Short PHY Preamble & $72 \mu \mathrm{s}$ & \\
\hline Long PHY Header & $48 \mu \mathrm{s}$ & \multirow{2}{*}{$4 \mu \mathrm{s}$} \\
\hline Short PHY Header & $24 \mu \mathrm{s}$ & \\
\hline Slot Time & \multicolumn{2}{|c|}{$20 \mu \mathrm{s}$} \\
\hline SIFS & \multicolumn{2}{|c|}{$10 \mu \mathrm{s}$} \\
\hline DIFS & \multicolumn{2}{|c|}{$50 \mu s$} \\
\hline PIFS & \multicolumn{2}{|c|}{$30 \mu s$} \\
\hline
\end{tabular}

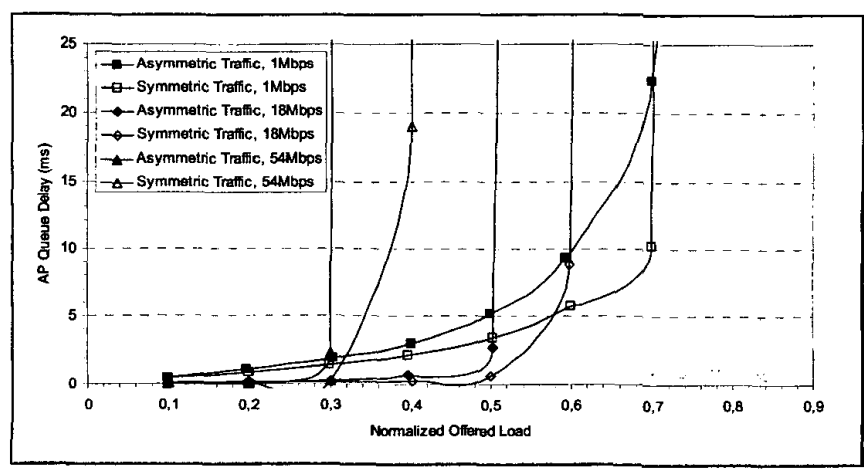

Fig. 1. AP queue delay

Finally, the throughput difference between symmetric and asymmetric traffic becomes lower as the transmission rate increases. For data rates up $18 \mathrm{Mbps}$, the saturation throughput values for symmetric and asymmetric traffic become closer, due to the decrease in the collision number and in the percentage of network backoff time difference between the asymmetric and the symmetric situation.

The saturation throughput decreases as the transmission data rate rises. For each packet transmission, the packet sent is composed of a PHY preamble, a PHY header and the data field. When using the IEEE $802.11 \mathrm{~b}$ physical layer, the long preamble and header are sent at $1 \mathrm{Mbps}$. On the other hand, considering the short format, the preamble is transmitted at 1 Mbps and the header at $2 \mathrm{Mbps}$. Finally, considering the ERP-OFDM physical layer, the preamble and header are sent at $6 \mathrm{Mbps}$. In this way, as the transmission data rate increases, the difference between data field transmission time and the preamble and header duration becomes higher and therefore the saturation throughput decreases. 


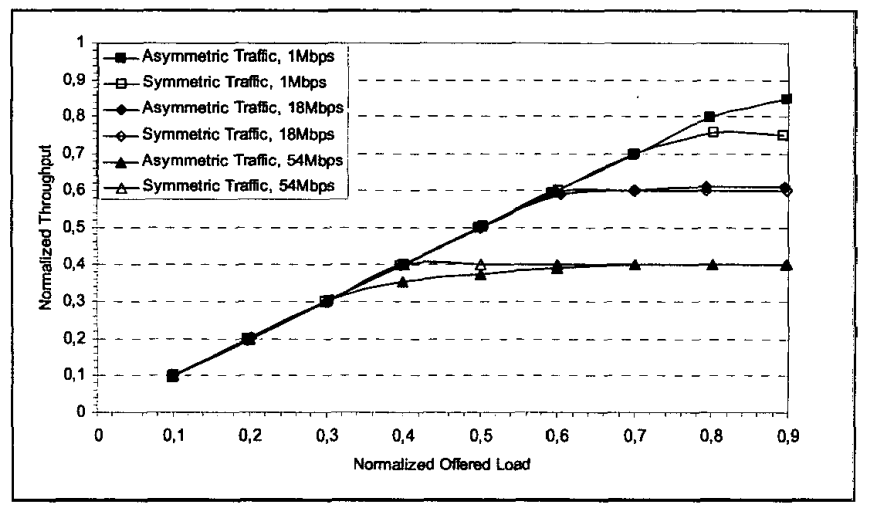

Fig. 2. Total throughput

\section{ENHANCEMENTS OF IEEE 802.11 UNDER ASYMMETRIC TRAFFIC}

In the previous section we have seen that under asymmetric traffic conditions, the AP queue delay is higher than under symmetric traffic, and, furthermore, the AP has more delay than the US. In order to improve the AP performance in presence of asymmetric traffic we have studied several methods based on increasing AP priority.

Firstly we reduce the AP minimum contention window value to $\mathrm{CW}_{\min }=8$ and let the $A P$ maximum contention window value at $\mathrm{CW}_{\max }=1024$. In this case we observe an important gain in the AP for different transmission rates (Fig. 3). For a transmission rate of $1 \mathrm{Mbps}$, with an offered load of 0.7 the AP queue delay decreases from $22 \mathrm{~ms}$ to $15 \mathrm{~ms}$, whereas in US the queue delay increases from $2 \mathrm{~ms}$ to $15 \mathrm{~ms}$.

Unfortunately, for a transmission rate of $1 \mathrm{Mbps}$ we obtain an important throughput reduction in heavy loaded systems (Fig 4), due to the consequently collision number growth and the increment of the percentage of network backoff time. This throughput decrease becomes less important as the transmission rate increases, because the growth in the collision number and in the percentage of network backoff time decreases.

Finally, the influence of the maximum contention window $\left(\mathrm{CW}_{\max }=1024\right.$ or $\left.\mathrm{CW}_{\max }=32\right)$ is minimal in both, $\mathrm{AP}$ queue delay and throughput.

As this easy method doesn't give the wished results, we propose other mechanisms of priorizing the AP transmission:

a) To include an initial backoff period in each user station data transmission.

b) To include a backoff period between two consecutive user station data transmissions.

c) To employ the IEEE 802.11e Arbitration Inter-Frame Space access time (AIFSD), instead of the DIFS time, which is determined by

$$
\text { AIFSD }[A C]=S I F S+A I F S[A C] \times \text { SlotTime , }
$$

where AIFS[AC] is an integer greater than zero, depending on its access category (AC). In order to give priority to the AP, we choose the following AP and user station AIFSD values

$$
\begin{aligned}
& A I F S D_{-} A P=S I F S+1 \times \text { SlotTime }=30 \mu \mathrm{s} \\
& A I F S D_{-} S U=S I F S+2 \times \text { SlotTime }=50 \mu \mathrm{s}
\end{aligned}
$$

d) To employ the IEEE 802.11e backoff increase function, where each AC has a different backoff increment function. The backoff time equation used is given by:

$$
\text { new } C W=((\text { old } C W+1) * P F[A C])-1,
$$

where $P F[A C]$ is the priority factor. We use an AP priority factor of $P F A P=2$ and a US $P F S U=6$.

e) Finally, we propose the combination of the mechanism explained in d) and the reduction of the AP minimum contention window value to $\mathrm{CW}_{\min }=8$.

The mechanisms explained in a), b), c) and d) do not provide any significant improvement respect the original case, and therefore we haven't plot them in this paper. Employing mechanisms c) and d), we only obtain a small reduction in the AP queue delay.

On the other hand, method e) provides changes in the protocol behaviour. Using method e), we get a reduction in the AP queue delay from $22 \mathrm{~ms}$ to $9 \mathrm{~ms}$, for a transmission rate of $1 \mathrm{Mbps}$ and an offered load of 0.7 . This reduction is greater than that obtained by reducing $\mathrm{AP} \mathrm{CW}_{\text {min }}$ value to 8 (Fig. 3).

Unfortunately, as expected, when we give more priority to the AP, user station's queue delay increases. 
Furthermore, employing method e) for a transmission rate of $1 \mathrm{Mbps}$ the throughput values (Fig. 4) obtained are very similar to the results presented in the original case. Moreover, for higher data rates, the throughput performance improves slightly.
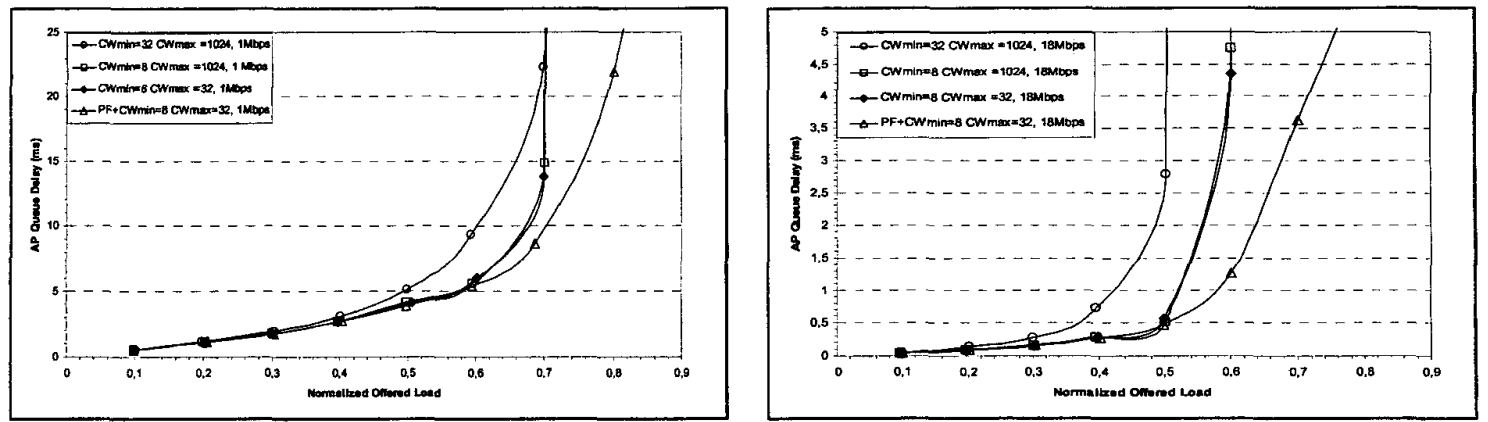

Fig. 3. AP queue delay for different AP contention window values and method e)
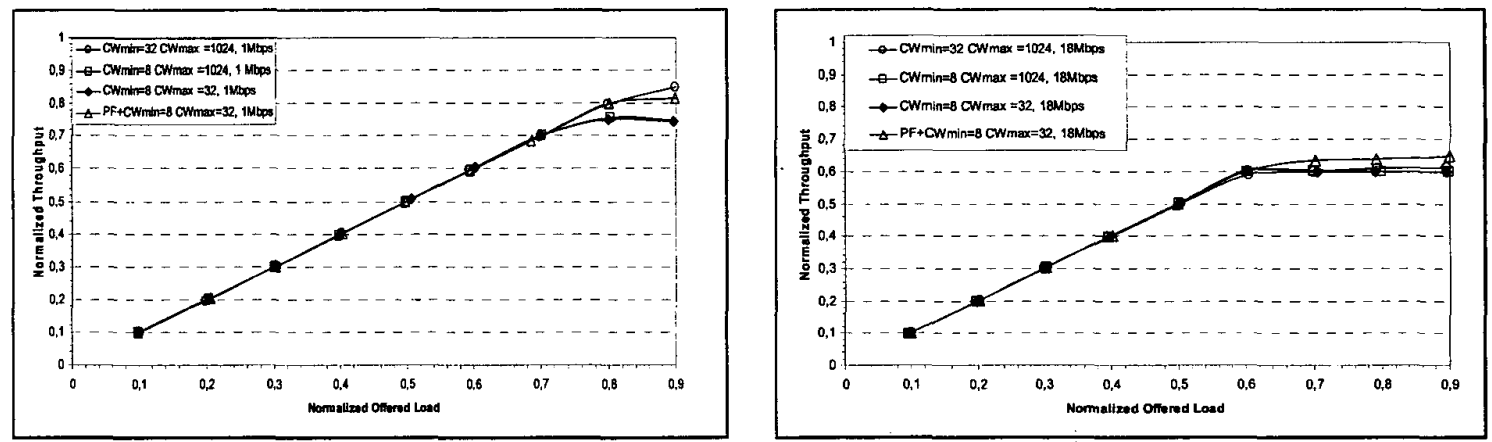

Fig. 4. Total throughput for different $A P$ contention window values and method e)

\section{CONCLUSIONS}

This paper studies the behaviour of IEEE 802.11 WLAN under conditions of asymmetric traffic, which is the more common case of WLAN used as access networks. We present different mechanisms in order to increase AP priority under an asymmetric traffic situation.

Method e), consisting on the combination of d) and the AP minimum contention window value reduction, offers the best AP performance in terms of queue delay, backoff time, transmission delay and throughput. On the other hand, mechanisms c) and d) only provide a small improvement in the AP performance, but offer better US performance than the method consisting on the AP CW $\mathrm{CW}_{\min }$ decrease.

\section{ACKNOWLEDGMENT}

This work was partially funded by the CICYT project TIC2003-01748.

\section{REFERENCES}

[1] G. Bianchi, "Performance analysis of the IEEE 802.11 Distributed Coordination Function", IEEE Journal on selected areas in communications, Vol. 18, No. 3, pp. 535-547, March 2000.

[2] F. Cali, M. Conti, E. Gregori. "Dynamic tuning of the IEEE 802.11 protocol to achieve a theoretical throughput limit", IEEE /ACM Transactions on networking, Vol. 8, No. 6, pp. 785-799, December 2000.

[3] S. Choi et al., "IEEE 802.11e contention-based channel access (EDCF) performance evaluation," IEEE International Conference on Communications 2003, Vol. 2, pp. 1151-1156, May 2003.

[4] 1. Aad, C. Castelluccia, "Differentiation mechanisms for IEEE 802.11", INFOCOM 2001, Vol. 1, pp. 209 - 218 , April 2001.

[5] D. Qiao, S. Choi, KG. Shin, "Goodput analysis and link adaptation for IEEE 802.11a Wireless LANs", IEEE Transactions on mobile computing, Vol. 1, No. 4, pp. 278-292, October 2002. 


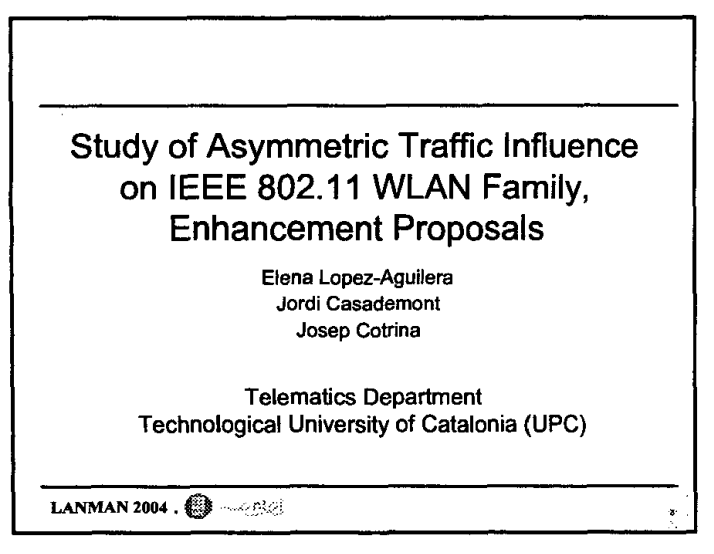

\begin{tabular}{l}
\hline Outline \\
\hline - Introduction \\
- IEEE $802.11 \mathrm{DCF}$ \\
- Simulation environment \\
- System evaluation under symmetric and \\
asymmetric traffic \\
- Enhancements of IEEE 802.11 under \\
asymmetric traffic \\
- Conclusions \\
\hline LANMAN 2004 $\mathrm{Ban}$ \\
\hline
\end{tabular}
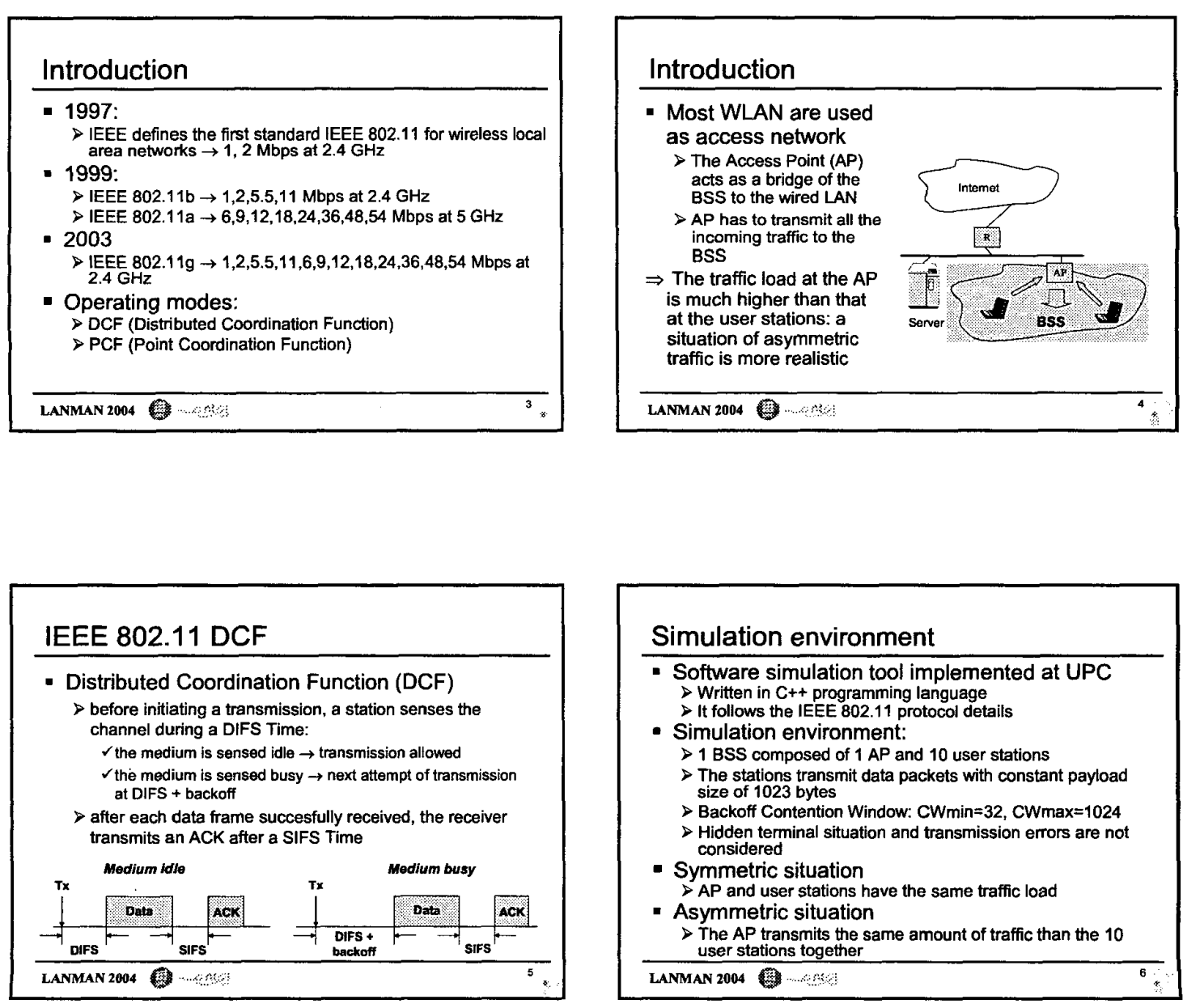

\section{Simulation environment}

- Software simulation tool implemented at UPC $>$ Written in $\mathrm{C}++$ programming language

$\rightarrow$ It follows the IEEE 802.11 protocol details

- Simulation environment:

$>1$ BSS composed of $1 \mathrm{AP}$ and 10 user stations

$>$ The stations transmit data packets with constant payload size of 1023 bytes

$>$ Backoff Contention Window: $C W m i n=32, C W m a x=1024$

$>$ Hidden terminal situation and transmission errors are not considered

- Symmetric situation

Symmetric situation

- Asymmetric situation

$>$ The AP transmits the same amount of traffic than the 10

user stations together

LANMAN 2004 

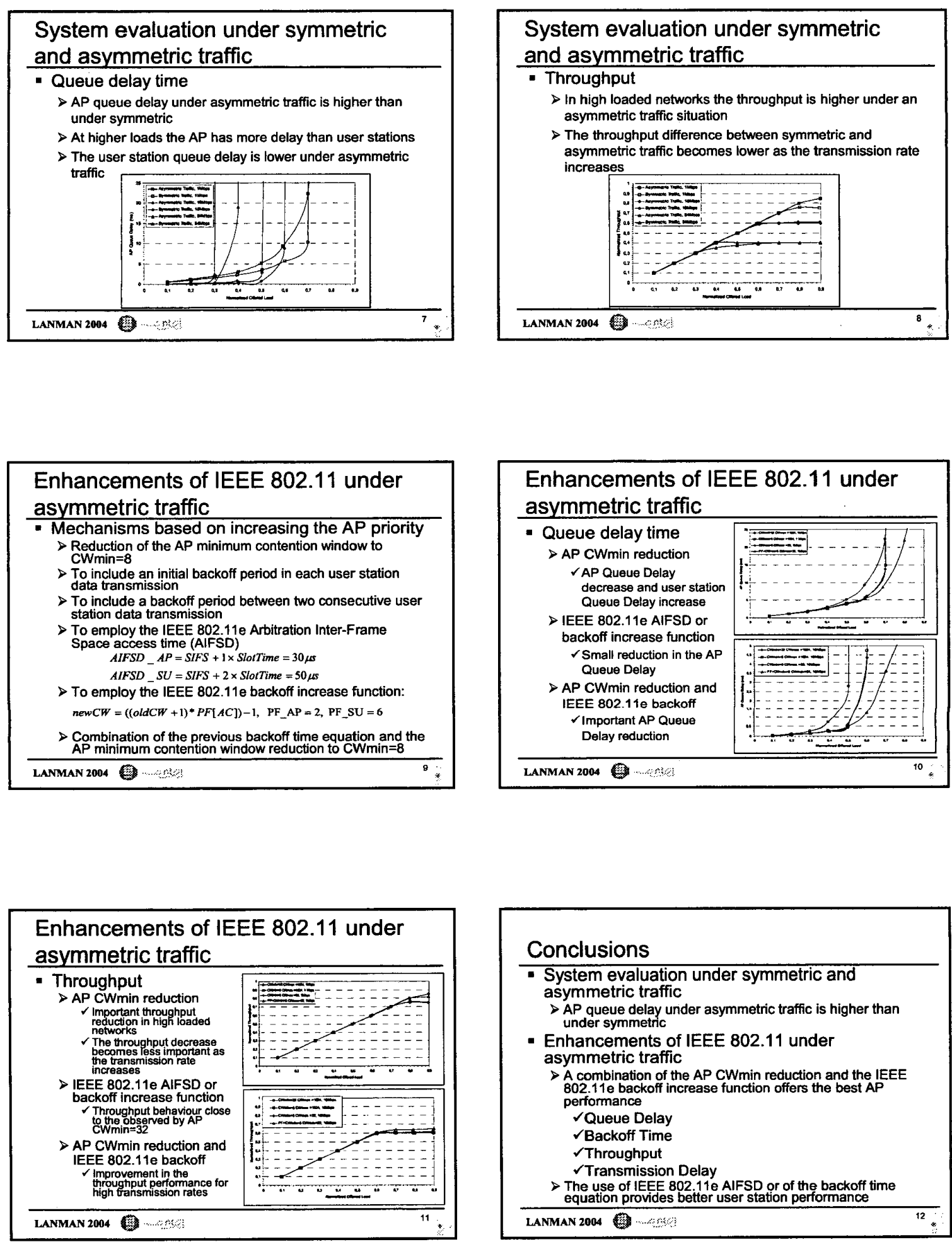

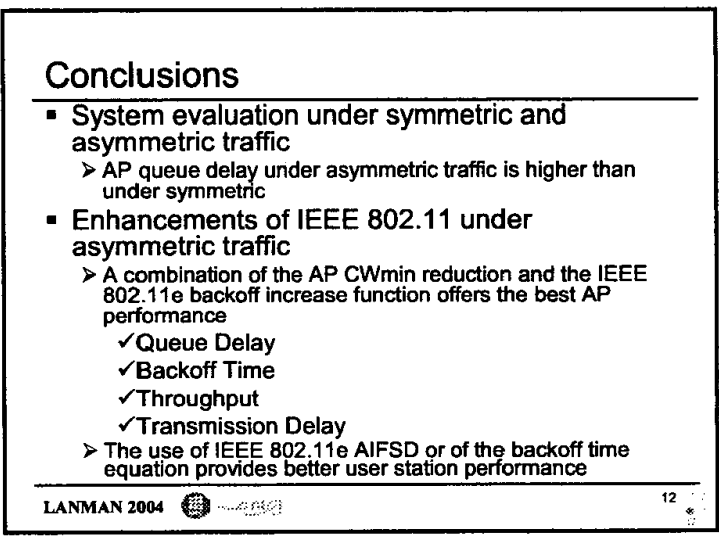

